\title{
Multiple Spontaneous Dissecting Aneurysms of the Anterior Cerebral and Vertebral Arteries
}

\author{
-Case Report- \\ Toshinori MAtsushige, Katsuzo KiYA, Hideki SAtoH, Tatsuya MizOue, \\ Kota KAGAWA, and Hayato ARAKI
}

Department of Neurosurgery, Hiroshima Prefectural Hospital, Hiroshima

\begin{abstract}
A 50-year-old woman presented with rare multiple dissecting aneurysms that appeared first in the anterior cerebral artery (ACA) and shortly afterwards in the vertebral artery (VA). She initially suffered sudden motor weakness in the left lower limb due to acute brain infarction. Angiography revealed diffuse string sign in the right ACA. Conservative treatment resulted in resolution of the deficits. Follow-up angiography performed 1 year later revealed recovery of the ACA stenosis. Fourteen days later, she complained of sudden headache and became comatose. Computed tomography showed diffuse subarachnoid hemorrhage. Angiography revealed a new right VA dissecting aneurysm involving the posterior inferior cerebellar artery (PICA). The orifice of the dissection was not apparent in the operative field and the dissection extended to the median. The patient underwent extracranial right VA ligation, clipping of the proximal PICA, and revascularization between the right occipital artery and distal PICA. Her postoperative course was uneventful and she was discharged without neurological deficits. VA dissecting aneurysms involving the PICA without evident orifice or extending over the median can be treated by extracranial ligation with clipping of the PICA, followed by revascularization.
\end{abstract}

Key words: dissecting aneurysm, subarachnoid hemorrhage, anterior cerebral artery, posterior inferior cerebellar artery, vertebral artery, revascularization

\section{Introduction}

Multiple spontaneous intracranial dissecting aneurysms are rare and constitute $10 \%$ of intracranial dissecting aneurysms in Japan. ${ }^{22,23)}$ Intracranial dissecting aneurysms in the supra- and infratentorial regions are even more rare, with only one such case. ${ }^{1)}$ Such aneurysms are an important cause of cerebrovascular disease. We treated a patient with multiple dissecting aneurysms in the anterior and posterior circulation that developed within 12 months. We also discuss treatment strategies for vertebral artery (VA) dissecting aneurysms involving the posterior inferior cerebellar artery (PICA).

\section{Case Report}

A 50-year-old woman with a history of hypertension was admitted to our institution with complaints of motor weakness in the left lower limb. She had no history of collagen diseases and her laboratory data was within normal limits. On admission, her consciousness was clear. Neurological examination found mild motor weakness of the left lower extremity. Diffusion-weighted and fluid-attenuated inversion recovery magnetic resonance imaging showed acute brain infarction in the territory of the right anterior cerebral artery (ACA) (Fig. 1). Cerebral angiography revealed diffuse string sign in the $A_{2}$ portion of the right ACA (Fig. 2). She was managed conservatively and discharged without neurological deficits.

One year later, follow-up three-vessel cerebral angiography showed normalization of the right ACA (Fig. 3). The right VA and PICA were completely visualized. Left vertebral angiography

Received April 26, 2004; Accepted November 25, 2004

Author's present address: T. Matsushige, M.D., Department of Neurosurgery, Matsue Red Cross Hospital, Matsue, Shimane, Japan. 

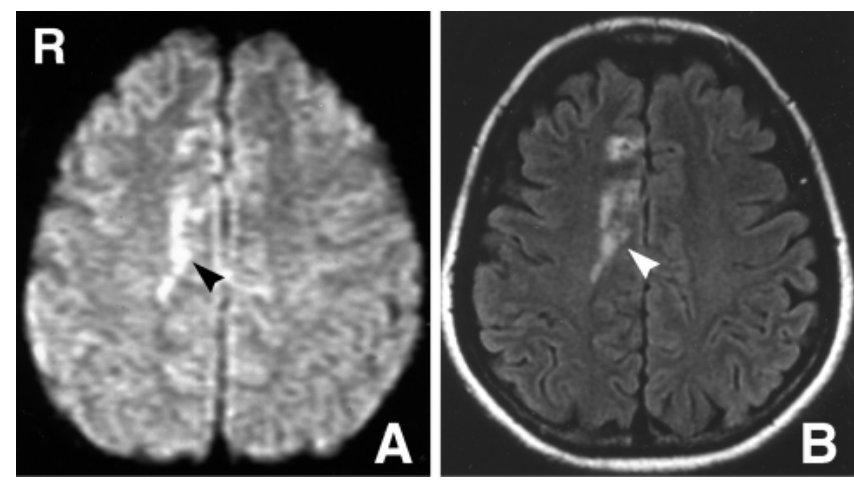

Fig. 1 Diffusion-weighted (A) and fluid-attenuated inversion recovery (B) magnetic resonance images showing acute brain infarction as a high-intensity area in the territory of the right anterior cerebral artery (arrowhead).

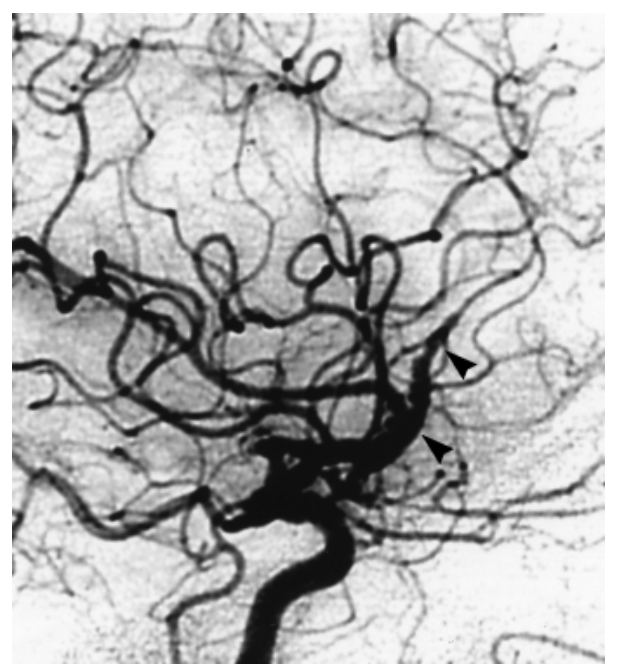

Fig. 2 Right carotid angiogram showing a dilatation of the right $A_{2}$ portion and diffuse string sign of the peripheral right anterior cerebral artery (arrowheads).

showed adequate reflux and no evidence of anomaly (Fig. 4). These findings confirmed the initial diagnosis of dissecting aneurysm of the right ACA. However, 2 weeks later, she again experienced sudden severe headache with consciousness deterioration. Computed tomography demonstrated diffuse subarachnoid hemorrhage (SAH) and emergency angiography revealed a dissecting aneurysm in the right VA involving the right PICA (Fig. 5). The area of dissection was suspected to extend from the fourth segment of the right VA. The distal VA including the dissection showed strong winding over the median. The right ACA was free of atypical

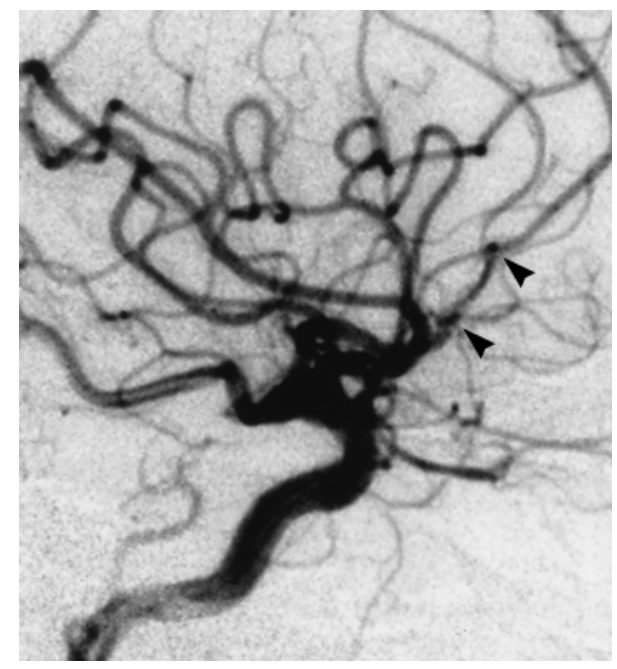

Fig. 3 Follow-up right carotid angiogram showing the normalized right anterior cerebral artery (arrowheads).

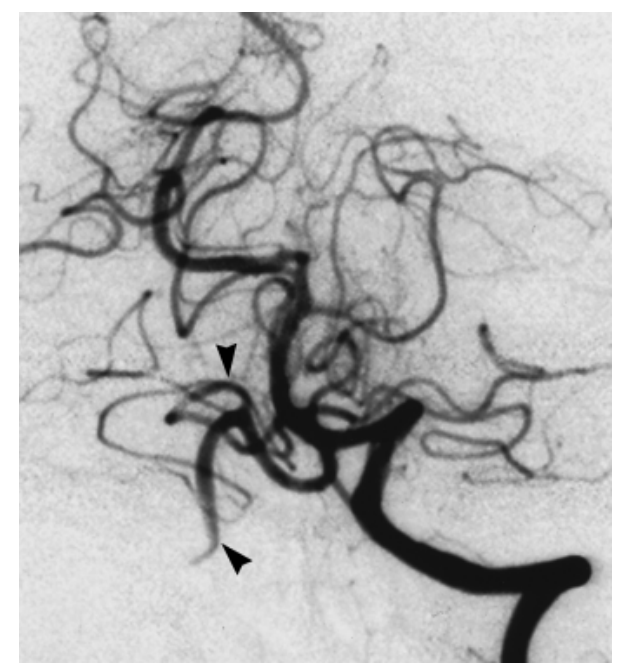

Fig. 4 Follow-up left vertebral angiogram showing the right vertebral artery and posterior inferior cerebellar artery with normal appearance (arrowheads). There is sufficient reflux to the right vertebral artery territory from the left vertebral artery.

changes. The patient became comatose due to aneurysm rerupture during the examination.

Emergent surgery was undertaken. The dissecting aneurysm involved the PICA and the fourth segment of the right VA, so the lateral suboccipital approach was adopted. Trapping of the ruptured aneurysm and revascularization were planned, but the right distal end of the dissection extended over the 
median and the proximal end was pale to the level of the lower cranial nerve. The distal portion of the dissected side beyond the median excluded the trapping procedure. Neither Doppler ultrasonographic imaging nor echography identified an orifice in the dissection. Therefore, extracranial proximal ligation of the patent VA was performed, with clipping of the PICA and revascularization between the occipital artery and the distal PICA. Doppler ultrasonographic imaging showed the dissecting site was free of flow. Follow-up angiography showed the distal VA contained a sponta-
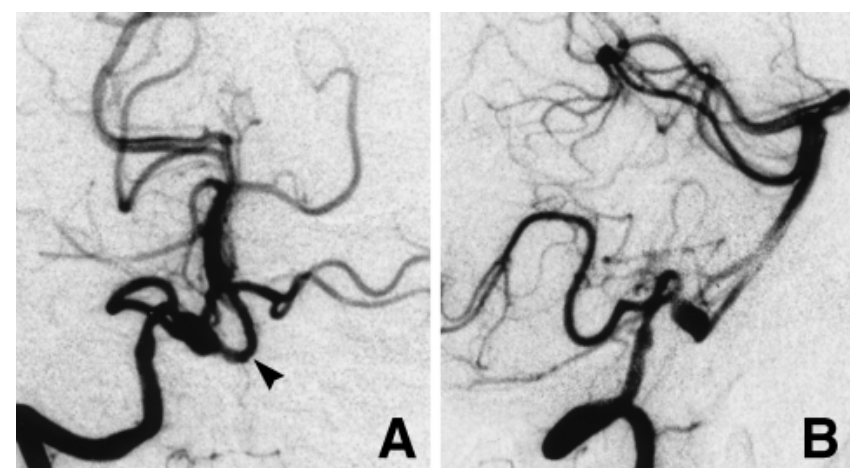

Fig. 5 Right vertebral angiograms, anteroposterior (A) and lateral views (B), obtained in the acute phase of subarachnoid hemorrhage. Note the extended pearl and string sign of the right vertebral artery involving the right posterior inferior cerebellar artery, and the distal portion of the right vertebral artery crossing over the median (A, arrowhead). neously formed thrombus at the blind end (Fig. 6). The patient was discharged on foot without neurological deficits 3 weeks after the operation.

\section{Discussion}

Only 14 cases of multiple spontaneous intracranial dissecting aneurysms have been reported. ${ }^{1-3,5,10,14}$, 16-19,25) The patients were nine males and five females aged from 33 to 65 years (mean 45.9 years). Multiple dissecting aneurysms can appear in the anterior and/or posterior circulation, but $80 \%$ of the reported multiple dissections were located in the posterior circulation involving the bilateral VAs, sometimes extending to the basilar artery.

Multiple dissecting aneurysms in the anterior circulation or supra- and infratentorial region are extremely rare. A patient first developed ACA dissecting aneurysm, and then de novo VA dissecting aneurysm was incidentally found 20 weeks after the onset of ACA dissection. ${ }^{1)}$ Another patient had multiple dissecting aneurysms that manifested as ACA ischemia about 6 weeks after initial middle cerebral artery dissection. ${ }^{10)}$ In our case, angiography obtained 2 weeks before the onset of SAH disclosed no remarkable anomaly of the right VA. The vessel showed adequate reflux, evidence that the right VA had sustained no mechanical damage during the earlier angiography.

The etiology of cerebral dissecting aneurysm can be classified into spontaneous and traumatic causes. A review of the potential precipitating events and risk factors of the dissection discovered no specific causes. $^{6)}$ Congenital factors, systematic collagen
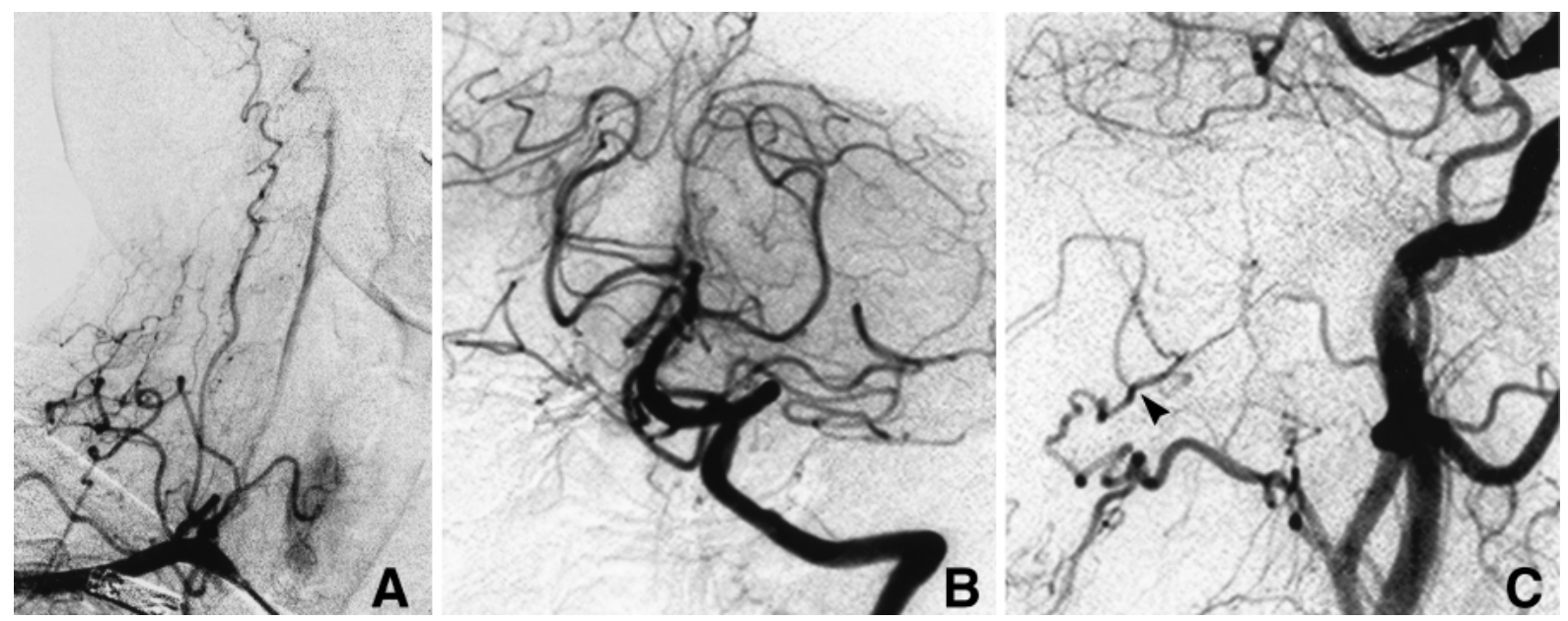

Fig. 6 Postoperative right (A) and left (B) vertebral angiograms demonstrating complete thrombosis of the site of dissection. Right carotid angiogram (C) showing the distal posterior inferior cerebellar artery (arrowhead) filling from the occipital artery. 
diseases, and angitis may be rarely associated with fragile tunica media or defect. Both our patient and reported patients with multiple dissecting aneurysms had no history of trauma, sports, or trivial injury during the period, or specific systematic diseases. The natural course of de novo dissecting aneurysms is also unclear. Some patients presented with secondary rupture of dissecting aneurysms after ischemic onset, or with asymptomatic dissecting aneurysms that were discovered in the course of long-term follow up. Aneurysms may be classified into four types based on their clinicopathological features. ${ }^{11,13)}$ However, the type may be difficult to discriminate from angiographic study and the time course of each type is still unknown. Our case suggests that, in contrast to saccular aneurysms, some types of dissecting aneurysms may suddenly occur and rupture, manifesting as SAH. Further case accumulation and evaluation are necessary for a better understanding of the etiology, natural history, and prognosis of intracranial dissecting aneurysms.

The posterior circulation includes major important branches and perforators supplying the brain stem, and the appropriate treatment of VA dissecting aneurysms involving the PICA remains unclear. The major problem with VA dissecting aneurysm involving the PICA is the poor outcome, with an overall morbidity reaching as high as $40 \% .^{25)}$ The extent of the dissection may complicate treatment efforts to prevent rupture and result in brain infarction.9) Surgical options for VA dissecting aneurysms involving the PICA include proximal clipping, ${ }^{5,8,12)}$ endovascular proximal occlusion, ${ }^{21,24)}$ endovascular internal trapping following balloon test occlusion,7) trapping with ${ }^{4,20)}$ or without ${ }^{2,12,24)}$ revascularization of the PICA, proximal clipping and PICA clipping with or without revascularization, and aneurysm body clipping. ${ }^{11)}$ Residual flow from the contralateral VA to the ipsilateral PICA ${ }^{1,5,8)}$ carries the risk of rebleeding if only proximal clipping is performed. Proximal clipping for VA dissections involving the PICA resulted in flow change in the pseudolumen due to reflux from the contralateral VA. ${ }^{15)}$ Therefore, the reflux flow into the pseudolumen must be assessed, despite technical difficulties in the acute stage, before proximal clipping is performed. Surgical treatment that sacrifices the PICA, i.e. trapping or proximal VA and PICA clipping without revascularization, induces cerebellar infarction in $50 \%$ of patients. ${ }^{13)}$ Treatment for VA dissecting aneurysm involving the PICA should be considered as revascularization. Treatment of our patient was successful because the portion of the dissected VA contained a spontane- ously formed thrombus at the blind end. We recommend proximal occlusion and PICA clipping with revascularization for patients with VA dissection if the orifice and exit zone cannot be clearly visualized in the course of surgery, or with maladjustment due to anatomical problems. If spontaneous thrombosis of the dissecting lesion can be induced, rerupture may be prevented.

\section{References}

1) Amagasaki K, Yagishita T, Yagi S, Kuroda K, Nishigaya $K$, Nukui $H$ : Serial angiography and endovascular treatment of dissecting aneurysms of the anterior cerebral and vertebral arteries: Case report. J Neurosurg 91: 682-686, 1999

2) Berger MS, Wilson CB: Intracranial dissecting aneurysms of the posterior circulation. Report of six cases and review of the literature. J Neurosurg 61: 882-894, 1984

3) Caplan LR, Baquis GD, Pessin MS, D'Alton J, Adelman LS, DeWitt LD, Ho K, Izukawa D, Kwan ES: Dissection of the intracranial vertebral artery. Neurology 38: 868-877, 1988

4) Durward QJ: Treatment of vertebral artery dissecting aneurysm by aneurysm trapping and posterior inferior cerebellar artery reimplantation. Case report. J Neurosurg 82: 137-139, 1995

5) Friedman AH, Drake CG: Subarachnoid hemorrhage from intracranial dissecting aneurysm. J Neurosurg 60: 325-334, 1984

6) Haldeman S, Kohlbeck FJ, McGregor M: Risk factors and precipitating neck movements causing vertebrobasilar artery dissection after cervical trauma and spinal manipulation. Spine 24: 785-794, 1999

7) Iihara K, Sakai N, Murao K, Sakai H, Higashi T, Kogure S, Takahashi JC, Nagata I: Dissecting aneurysms of the vertebral artery: a management strategy. J Neurosurg 97: 259-267, 2002

8) Kitanaka C, Morimoto T, Sasaki T, Takakura K: Rebleeding from vertebral artery dissection after proximal clipping. Case report. J Neurosurg 77: 466-468, 1992

9) Kitanaka C, Sasaki T, Eguchi T, Teraoka A, Nakane M, Hoya K: Intracranial vertebral artery dissections: clinical, radiological features, and surgical considerations. Neurosurgery 34: 620-626, 1994

10) Kurino M, Yoshioka S, Ushio Y: Spontaneous dissecting aneurysms of anterior and middle cerebral artery associated with brain infarction: a case report and review of the literature. Surg Neurol 57: 428-436, 2002

11) Mizutani $\mathrm{T}$ : [Surgical consideration for cerebral dissecting aneurysms]. Surgery for Cerebral Stroke 30: 424-428, 2002 (Jpn, with Eng abstract)

12) Mizutani $T$, Aruga $T$, Kirino $T$, Miki $Y$, Saito $I$, Tsuchida T: Recurrent subarachnoid hemorrhage from untreated ruptured vertebrobasilar dissecting aneurysms. Neurosurgery 36: 905-911, 1995 
13) Mizutani T, Kojima H: Clinicopathological features of non-atherosclerotic cerebral arterial trunk aneurysms. Neuropathology 20: 91-97, 2000

14) Mizutani T, Miki Y, Kojima H, Suzuki H: Proposed classification of nonatherosclerotic cerebral fusiform and dissecting aneurysms. Neurosurgery 45: 253-259, 1999

15) Nakatomi H, Nagata K, Kawamoto S: Persistent patent pseudolumen of ruptured dissecting aneurysm involving the posterior inferior cerebellar artery after proximal clipping. Acta Neurochir (Wien) 141: 533-536, 1999

16) Otawara Y, Ogasawara K, Ogawa A, Kogure T: Dissecting aneurysms of the bilateral vertebral arteries with subarachnoid hemorrhage: report of three cases. Neurosurgery 50: 1372-1374, 2002

17) Otawara Y, Suzuki M, Abe M, Tomizuka N, Ogawa A: Dissecting aneurysms of the anterior cerebral artery and accessory middle cerebral artery. Case report. Neurosurg Rev 20: 145-148, 1997

18) Redekop G, TerBrugge K, Willinsky R: Subarachnoid hemorrhage from vertebrobasilar dissecting aneurysm treated with staged bilateral vertebral artery occlusion: the importance of early follow-up angiography: technical case report. Neurosurgery 45: 1258-1262, 1999

19) Sakamoto $S$, Inagawa $T$, Ikawa $F$, Kawamoto $H$, Ohbayashi N: Anterior cerebral artery dissections manifesting as cerebral hemorrhage and infarction, and presenting as dynamic angiographical changes - Case report. Neurol Med Chir (Tokyo) 42: 250-254, 2002

20) Takikawa S, Kamiyama H, Nomura M, Abe H, Saitoh $\mathrm{H}$ : [Vertebral dissecting aneurysm treated with trapping and bilateral posterior inferior cerebellar artery side-to side anastomosis; case report]. No Shinkei Geka 19: 571-576, 1991 (Jpn, with Eng abstract)

21) Yamada M, Miyasaka K, Kurata A, Yada K, Kitanaka T, Ohwada T, Takagi H: [Problem in the treatment of intracranial dissecting vertebral aneurysms]. Surgery for Cerebral Stroke 22: 41-46, 1994 (Jpn, with Eng abstract)

22) Yamaura A, Ono J, Okimura Y, Fukuda K, Miyata A: [Analysis of nontraumatic intracranial arterial dissections: Carotid lesion vs. vertebrobasilar lesion]. Surgery for Cerebral Stroke 21: 341-346, 1993 (Jpn, with Eng abstract)

23) Yamaura A, Yoshimoto T, Hashimoto N, Ono J: [Nationwide study of nontraumatic intracranial arterial dissection: Clinical features and outcome]. Surgery for Cerebral Stroke 26: 79-86, 1998 (Jpn, with Eng abstract)

24) Yasui T, Komiyama M, Nishikawa M: Subarachnoid hemorrhage from vertebral artery dissecting aneurysms involving the origin of the posteroinferior cerebellar artery: report of two cases and review of the literature. Neurosurgery 46: 196-201, 2000

25) Yasui T, Sakamoto H, Kishi H, Komiyama M, Iwai Y, Yamanaka K, Nishikawa M: Bilateral dissecting aneurysms of the vertebral arteries resulting in subarachnoid hemorrhage: case report. Neurosurgery 42: 162-164, 1998

Address reprint requests to: T. Matsushige, M.D., Department of Neurosurgery, Matsue Red Cross Hospital, 200 Horo-machi, Matsue, Shimane 690-8506, Japan. e-mail: h-k1122@hicat.ne.jp 